\begin{tabular}{|l|l|}
\hline $\begin{array}{l}\text { Instituto de } \\
\text { Geriatria e Gerontologia }\end{array}$ & $\begin{array}{l}\text { Pan American Journal of Aging Research } \\
\text { PAJAR, Porto Alegre, v. 9, p. 1-4, jan.-dez. } 2021 \\
\text { ISSN-L: 2357-9641 }\end{array}$ \\
\hline http://dx.doi.org/10.15448/2357-9641.2021.1.41087 & \\
\cline { 1 - 2 }
\end{tabular}

EDITORIAL

\title{
Healthy aging during the COVID-19 pandemic
}

Gisele Hansel ${ }^{1}$

orcid.org/0000-0001-5048-1293

gisele.hansel@pucrs.br

\section{Douglas Kazutoshi}

\section{Sato ${ }^{1}$}

orcid.org/0000-0002-7695-6020

douglas.sato@pucrs.br

Received on: Jun. 07th, 2021 Approved on: Jun. 08th, 2021.

\section{(c) (i)}

Artigo está licenciado sob forma de uma licença Creative Commons Atribuição 4.0 Internacional.
On December 31st, 2019, the World Health Organization (WHO) was alerted of several cases of pneumonia in Wuhan, China. Since then, the severe acute respiratory syndrome coronavirus-2 (SARS-CoV-2) agent was identified as a new and highly contagious respiratory pathogen that causes a disease called the 2019 coronavirus disease (COVID-19). The high spread rate and the lack of effective treatment for the disease have put the world community in the Public Health Emergency of International Importance - the highest alert level of WHO, as provided for the International Health Regulations $(1,2)$.

Although the majority of patients develops mild to moderate COVID-19, we experienced severe cases with acute respiratory distress syndrome (ARDS) requiring a high number of intensive care units. The major risk factors for severe COVID-19 includes age, obesity, smoking, and comorbid chronic conditions such as hypertension and diabetes. The age itself is likely to be the most significant risk factor for severe COVID-19 (1). The elderly people accounts for only $9 \%$ of the global population, but this group represents more than $80 \%$ of COVID-19 related deaths (3).

The WHO recommendations to decrease the SARS-CoV-2 spreading include wearing a mask, washing hands frequently, keeping rooms well ventilated, and social distancing (1). On the other hand, social interaction is an important factor for the life quality of the elderly, helping with their well-being, reducing levels of stress and depression, and promoting positive cognitive effects. Therefore, we need to balance the adequate social distancing and reorganize activities important to the quality of life (4).

The COVID-19 pandemic challenged the way to think about healthy aging. Stimulating elderly people socially and interaction with other people - relatives and friends-, as well as perform physical activities in large groups were discouraged to avoid SARS-CoV-2 spreading. Today, the major challenge for healthy aging during the COVID-19 is to find the best balance between social well-being, mental health and safety.

The clinical aspects and biological markers have not yet been fully identified and, so far, do not explain why aging itself is a risk factor for severe forms of COVID-19. Therefore, we need to assess the influence of biological aging (not only chronological age) in the outcomes for SARS-CoV-2 infection. If the immunological and inflammatory response is abnormal 
in this population, we may provide guidance to reduce the risk of severe cases and propose public health strategies to this population (5).

Amid so several doubts, some questions need to be addressed to understand the effect of COVID-19 severity on the elderly: 1) Why would aging be a risk factor itself for SARSCoV-2 infection? 2) Could aging change the immunogenicity of vaccines developed for SARSCoV-2? 3) Which are the precautions needed after vaccination for the elderly? Moreover, the most important, 4) How to balance healthy aging and social distancing during the pandemics?

The aging changes physiological processes alter the functionality of various tissues, organs, and systems. The changes are multifactorial and heterogeneous, varying from person to person, which makes it difficult to recognize a single factor to explain the development of several diseases and vulnerability to infections $(6,7)$. The outcome of any infectious disease depends on both innate and adaptive immune responses. The innate immune mechanisms are responsible for pathogen protein recognition through cellular receptors expressed in dendritic cells and macrophages, followed by the production of signaling molecules such as cytokines, tumor necrosis factor, and chemokines which signalize neutrophils and other immune cells to the site of infection (8). The adaptive immune response is initiated after the pathogen antigen presentation to CD4 helper $T$ cells and CD8 cytotoxic T cells, which attack infected cells per se and induce B cells to produce high-affinity antibodies to control the infection and retain long-lasting immune memory. These immune mechanisms are complex and need a fine-tuned orchestra to eliminate the pathogen without causing damage to the organism $(3,8)$.

The immune system changes are one of the hallmarks of the aging process. Immunosenescence, defined as a gradual decrease in immune function, promoting deficient humoral and cellular response, and the chronic low-grade inflammation, known as 'inflammaging', are considered key features for immune dysfunction in the elderly (3.5). Moreover, abnormal immune responses may induce uncontrolled inflammation and autoimmune disorders. Although the relationship between SARS-CoV-2 infection and immunosenescence is not fully understood, it seems to determine not only the susceptibility to other infections but also the clinical outcomes. Studies have shown basal hypersecretion of pro-inflammatory cytokines during aging affects several mechanisms such as tissue regeneration, repair, and homeostasis. Therefore, inflammaging may alter the body response to SARS-CoV-2 with abnormal innate and adaptive immune response activations. This results in overproduction of pro-inflammatory molecules, known as "cytokine storm" that may result in ARDS and other life-threatening situations seen in COVID-19.

This abnormal immune response, besides causing a delay in the primary defense against the pathogen, generates exacerbated cellular damage during the SARS-CoV2 infection. $(2,5)$. Few studies have shown that the increase of neutrophils-to-lymphocytes ratio, as well as the increase of IL-6 production, were correlated to poor prognosis for COVID-19 $(5,8)$. A study with COVID-19 patients showed that restored lymphocyte counts predicted recovery, while lymphopenia persists in non-survivors (9). These findings emphasize the importance of cell-mediated immunity and its direct impact on clinical prognosis. The basal changes that occur during biological aging such as modifications in the immune cell profile and increased proinfammatory cytokines production could predispose to severe COVID-19 $(5,8)$.

Until now, the vaccine seems to be the best intervention against the SARS-CoV-2 infection. However, in the elderly population, the efficacy may be affected due to the changes before mentioned physiological changes to the immune system. Thus, the main question after vaccine development is whether the elderly can effectively develop a robust and durable response to COVID-19 vaccines. In several studies, the markedly reduced vaccine efficacy in elderly has been attributed to adaptive immunosenescence on $\mathrm{T}$ lymphocytes and decreased humoral immunity (10-12). 
The immunological response to vaccines in the elderly is heterogeneous. A study with yellow fever vaccination revealed that elderly people generate lower levels of neutralizing antibodies compared to young adults and the $\mathrm{CD} 4^{+}$and CD8+ T effector cells were much lower than controls. The vaccination in the elderly is usually aimed to increase preexisting immunity from previous infections and vaccinations. The booster dosing causes a reduction in the severity of the disease but does not eliminate the possibility of infection, as experienced with influenza virus $(10,12)$.

The vaccines against COVID-19 have been developed in a never-seen speed with biotechnologies that are different from most of currently available vaccines for other diseases. Nowadays, more than 45 vaccines for SARS-CoV-2 are already being used for vaccinating the world population, or are under clinical trial studies. The technologies used are the most varied, including live attenuated virus, inactivated virus, viral vector, virus-like particles, subunit, and nucleic acid vaccines (10). Several studies are analyzing the different technologies to drive the effectiveness of those vaccines, especially in populations with a deficient immune response. So far, there is no evidence that one specific technology is better than another and the utility of different vaccine types in the same person to boost the immune response requires further evaluation.

A Brazilian study evaluated the early impact of immunization against COVID-19 on deaths among elderly people. The data showed the prevalence of death in the elderly over 80 years of age dropped from $25-30 \%$ to $15 \%$ after immunization, despite the predominance of Brazil's P1 coronavirus variant, which has a high spread rate and causes more aggressive disease (13). Importantly, lifestyle factors such as a balanced diet and physical activity are important factors to improve the effects of vaccines in the elderly. For example, cardiovascular exercises result in improved antibody responses to influenza vaccination with higher seroprotection after 24 weeks (14), suggesting the benefits of physical activity and adequate nutrition in the pandemic period.
We conclude that healthy aging has been challenging during the COVID-19 pandemics. The required social distancing to avoid SARS-CoV-2 infection heavily impacted the mental health and other activities so beneficial for elderly people. Beside other risk factors, the inflammaging may ease aberrant immune responses causing severe COVID-19 and increased mortality. Moreover, the vaccines for SARS-CoV-2 could require additional boosting doses, changes in the formulation, mixed vaccination strategies for this age group, as well as continuous monitoring for their susceptibility to the new variants of the virus. Thus, research on the impact of these factors in the COVID-19 and vaccines may result in a biological footprint for a personalized medicine with tailored preventive and therapeutic measures for each individual. This may allow low-risk elderly individuals to resume their social activities after vaccination, while continuing the other safety measures.

\section{References}

1. World Health Organization. WHO. Coronavirus. 2021 (May, 24 2021). Retrived from https://www.who.int/ health-topics/coronavirus\#tab=tab_1

2. Chen Y, Klein SL, Garibaldi BT, Li H, Wu C, Osevala NM, et al. Aging in COVID-19: Vulnerability, immunity and intervention. Ageing Res Rev [Internet]. 2021;65(August 2020):101205. Available from: https://doi.org/10.1016/j. arr.2020.101205

3. Biswas A, Mandal RS, Chakraborty S, Maiti G. Tapping the immunological imprints to design chimeric SARS-CoV-2 vaccine for elderly population. Int Rev Immunol [Internet]. 2021;0(0):1-16. Available from: http://www. ncbi.nlm.nih.gov/pubmed/33978550

4. Margarete Diprat Trevisan Patricia Morsch, Lopes DGC, Oliveira GG de, Bós ÂJG. Association between quality of life and activities developed by older adults from the Rio Grande do Sul state. Pajar. 2017:5(2):47-54.

5. Nehme J, Borghesan M, Mackedenski S, Bird TG, Demaria M. Cellular senescence as a potential mediator of COVID-19 severity in the elderly. Aging Cell. 2020;19(10):1-14.

6. Sarzi-Puttini P, Giorgi V, Sirotti S, Marotto D, Ardizzone S, Rizzardini G, et al. COVID-19, cytokines and immunosuppression: what can we learn from severe acute respiratory syndrome? Clin Exp Rheumatol. 2020;38(2):337-42.

7. McGonagle D, Sharif K, O'Regan A, Bridgewood C. The Role of Cytokines including Interleukin-6 in COVID-19 induced Pneumonia and Macrophage Activation Syndrome-Like Disease. Autoimmun Rev. 2020;19(6). 
8. Bajaj V, Gadi N, Spihlman AP, Wu SC, Choi CH, Moulton VR. Aging, Immunity, and COVID-19: How Age Influences the Host Immune Response to Coronavirus Infections? Front Physiol. 2021;11(January):1-23.

9. Chen R, Sang L, Jiang M, Yang Z, Jia N, Fu W, et al. Longitudinal hematologic and immunologic variations associated with the progression of COVID-19 patients in China. J Allergy Clin Immunol. 2020;146(1):89-100.

10. Connors J, Bell MR, Marcy J, Kutzler M, Haddad EK. The impact of immuno-aging on SARS-CoV-2 vaccine development. GeroScience. 2021;(March 2020):31-51.

11. Tenforde MW, Olson SM, SelfWH, Talbot HK, Lindsell CJ, Steingrub JS, et al. Effectiveness of Pfizer-BioNTech and Moderna Vaccines Against COVID-19 Among Hospitalized Adults Aged $\geq 65$ Years - United States, January-March 2021. MMWR Morb Mortal Wkly Rep [Internet]. 2021;70(18):674-9. Available from: http:// www.ncbi.nlm.nih.gov/pubmed/33956782

12. Lisa Müller, Marcel Andrée, Wiebke Moskorz, Ingo Drexler, Lara Walotka, Ramona Grothmann, Johannes Ptok, Jonas Hillebrandt, Anastasia Ritchie, Denise Rabl, Philipp Niklas Ostermann, Rebekka Robitzsch, Sandra Hauka, Andreas Walker, Christopher Menne, Ralf HS. Age-dependent immune response to the Biontech/Pfizer BNT162b2 COVID-19 vaccination. medRxiv [Internet]. 2021:2021.03.03.21251066. Available from: https://Www. medrxiv.org/content/10.1101/2021.03.03.21251066v1

13. Victora CG, Castro MC, Gurzenda S, Barros AJD. Estimating the early impact of immunization against COVID-19 on deaths among elderly people in Brazil: analyses of secondary data on vaccine coverage and mortality. medRxiv [Internet]. 2021;2021.04.27.21256187. Available from: http://medrxiv.org/content/early/2021/04/30/2021.04.27.21256187.abstract

14. Woods JA, Keylock KT, Lowder T, Vieira VJ, Zelkovich W. Dumich S, et al. Cardiovascular exercise training extends influenza vaccine seroprotection in sedentary older adults: The immune function intervention trial. J Am Geriatr Soc. 2009:57(12):2183-91.

\section{Gisele Hansel}

Post-doctoral fellow in Medicine and Health Sciences (CAPES-Print). Postgraduate Program in Medicine and Health Sciences, Pontifical Catholic University of Rio Grande do Sul (PUCRS), Porto Alegre, RS, Brazil.

\section{Douglas Kazutoshi Sato}

Neurologist Physician; Professor at the School of Medicine; Director of the Institute of Geriatrics and Gerontology; Researcher at the Brain Institute of Rio Grande do Sul (InsCer); Pontifical Catholic University of Rio Grande do Sul (PUCRS), Porto Alegre, RS, Brazil.

\section{Disclosures}

Authors reports no disclosures related to this article.

\section{Corresponding author:}

Douglas Kazutoshi Sato

Av. Ipiranga, 6690

Prédio 63, Instituto do Cérebro do Rio Grande do Sul CEP 90610-000 - Bairro Jardim Botânico

Porto Alegre/RS 